\title{
Photoacoustic molecular imaging of small animals in vivo
}

\author{
Xueyi Xie, Meng-Lin Li, Jung-Taek Oh, Geng Ku, Wei \\ Wang, et al.
}

Xueyi Xie, Meng-Lin Li, Jung-Taek Oh, Geng Ku, Wei Wang, Chun Li, Sergiu Similache, Gina F. Lungu, George Stoica, Lihong V. Wang, "Photoacoustic molecular imaging of small animals in vivo," Proc. SPIE 6086, Photons Plus Ultrasound: Imaging and Sensing 2006: The Seventh Conference on Biomedical Thermoacoustics, Optoacoustics, and Acoustooptics, 608606 (6 March 2006); doi: 10.1117/12.645121

SPIE. Event: SPIE BiOS, 2006, San Jose, California, United States 


\title{
Photoacoustic molecular imaging of small animals in vivo
}

\author{
Xueyi Xie ${ }^{*}$, Meng-Lin $\mathrm{Li}^{1^{*}}$, Jung-Taek $\mathrm{Oh}^{1 *}$, Geng $\mathrm{Ku}^{1}$, Wei Wang ${ }^{2}$, \\ Chun $\mathrm{Li}^{2}$, Sergiu Similache ${ }^{3}$, Gina F. Lungu ${ }^{3}$, George Stoica ${ }^{3}$, and Lihong V. Wang ${ }^{\text {*** }}$ \\ 1. Optical Imaging Laboratory, Department of Biomedical Engineering, Texas A\&M University, \\ College Station, TX \\ 2. Division of Diagnostic Imaging, The University of Texas M. D. Anderson Cancer Center, \\ Houston, TX \\ 3. Department of Veterinary Pathobiology, Texas A\&M University, College Station, TX \\ * These authors contributed equally to this work \\ ** Corresponding author: email: lwang@tamu.edu; tel: 979-847-9040; fax: 979-845-4450
}

\begin{abstract}
Molecular imaging is a newly emerging field in which the modern tools of molecular and cell biology have been married to state-of-the-art technologies for noninvasive imaging. The study of molecular imaging will lead to better methods for understanding biological processes as well as diagnosing and managing disease. Here we present noninvasive in vivo spectroscopic photoacoustic tomography (PAT)-based molecular imaging of $\alpha v \beta 3$ integrin in a nude mouse U87 brain tumor. PAT combines high optical absorption contrast and high ultrasonic resolution by employing short laser pulses to generate acoustic waves in biological tissues through thermoelastic expansion. Spectroscopic PAT-based molecular imaging offers the separation of the contributions from different absorbers based on the differences in optical absorption spectra among those absorbers. In our case, in the near infrared (NIR) range, oxy-heamoglobin $\left(\mathrm{O}_{2} \mathrm{Hb}\right)$, deoxy-heamoglobin $(\mathrm{HHb})$ and the injected $\alpha \mathrm{v} \beta 3$-targeted peptide-ICG conjugated NIR fluorescent contrast agent are the three main absorbers. Therefore, with the excitation by multiple wavelength laser pulses, spectroscopic PAT-based molecular imaging not only provides the level of the contrast agent accumulation in the U87 glioblastoma tumor, which is related to the metabolism and angiogenesis of the tumor, but also offers the information on tumor angiogenesis and tumor hypoxia.
\end{abstract}

Keywords: Photoacoustic tomography, molecular imaging, NIR contrast agent, spectroscope

\section{INTRODUCTION}

The development of noninvasive in vivo molecular imaging techniques has been an area of great clinical interest. Initially driven by in situ reporter imaging technology, the study of molecular imaging should lead to better methods for studying biological processes as well as diagnosing and managing disease. The three common noninvasive molecular imaging technologies that have been developed more-or-less in parallel, are: $(a)$ MR imaging, ${ }^{1}(b)$ nuclear imaging (quantitative autoradiography, gamma camera and PET), ${ }^{2}$ and $(c)$ in vivo optical imaging of small animals. ${ }^{3} \mathrm{~A}$ comprehensive review of these techniques is beyond the scope of this paper, but excellent reviews can be found in the literature. ${ }^{4}$ Among these technologies, in vivo optical imaging faces the greatest challenge, which is the highly scattering nature of the biological tissues that significantly degrades the resolution. Photoacoustic tomography (PAT) is an emerging imaging modality that overcomes the scattering drawback and takes advantage of the merits and most compelling features of both optics and ultrasound, providing both high optical contrast and good ultrasound resolution. ${ }^{5}$ PA signals are induced by pulsed laser illumination. When laser energy is absorbed by biological tissues, the resulting thermal expansion of the tissues generates ultrasonic waves. The ultrasonic waves can be detected by an ultrasonic transducer and then used to reconstruct the optical absorption distribution inside the tissues. PAT has been shown to be 
a promising tool for biomedical applications, such as the monitoring of oxygenation in blood vessels, epidermal melanin measurement, angiography, and breast tumor detection. ${ }^{6}$

Here we present a noninvasive in vivo spectroscopic PAT-based molecular imaging technology. Our current in vivo molecular imaging strategy involves the coupling of a $\alpha v \beta 3$ integrin with a complimentary integrin $\alpha v \beta_{3}-$ targeted peptide-ICG conjugated NIR fluorescent contrast agent. ${ }^{7}$ The $\alpha_{v} \beta_{3}$-targeted peptide-ICG conjugated NIR fluorescent contrast agent was systematically administrated through the tail vein into the nude mice which had been implanted with a U87 glioma tumor within the brain. Imaging the level of contrast agent uptake provides direct information related to the metabolism and angiogenesis of the tumor. Spectroscopic PAT-based molecular imaging, which is based on the differences in the optical absorption spectra among the oxyhemoglobin $\left(\mathrm{O}_{2} \mathrm{Hb}\right)$, deoxyhemoglobin $(\mathrm{HHb})$ and the contrast agent, can be used to image not only the contrast agent uptake but also the total hemoglobin concentration $(\mathrm{HbT})$ and tumor hypoxia.

\section{MATERIALS AND METHODS}

\subsection{Animal Protocols}

All of the performed experimental animal procedures on the nude mice were approved by the University Laboratory Animal Care Committee of Texas A\&M University and followed the guidelines of the United States National Institutes of Health (Guide for the Care and Use of Laboratory Animals, NIH Publication No. 86-23, revised 1985. US Government Printing Office, Washington DC).

U87 glioblastoma tumour cells were used for implantation into young adult immunocompromised nude mice (Harlan, Co.). The cell line is maintained in Dulbecco's Modified Eagle Medium (DMEM) with 10000 Units/ml Penicillin and 10\% Fetal Bovine Serum (Gibco, Inc.). Stereotactic implantation of the U87 glioblastoma tumour cells was performed on the nude mice, which weighted about 20 grams, under full anaesthesia using a mixture of ketamine, $87 \mathrm{mg} / \mathrm{kg}$ (Ketaset, Forth Dodge Animal Health) and xylazine, $13 \mathrm{mg} / \mathrm{kg}$ (AnaSed, Lloyd Laboratories). The cells $\left(1 \times 10^{6}\right)$ were inoculated intracranially into the caudate nucleus at a $3 \mathrm{~mm}$ depth from the mouse head surface with a volume of $7 \mu 1$ by using a $10 \mu 1$ Hamilton syringe. ${ }^{8}$

The molecular contrast agent was intravenously administrated into the nude mice through the tail vein. In vivo fluorescence and PAT images were acquired 20 hours after the injection. The nude mouse body temperature was controlled at $37^{\circ} \mathrm{C}$ using a water heating pad. The health status, pulse rate and the global arterial blood oxygenation of the mouse were monitored through the entire spectroscopic photoacoustic tomography experiment using a pulse oximeter (Model 8600, Nonin Medical, Inc.) with a detector clamped on the right back paw of the nude mouse. After the experiments, the mice were sacrificed to obtain brain tissue sections/slides. One third of the slides were imaged using an Odyssey fluorescence microscanner at $800 \mathrm{~nm}$ to verify the uptake of the molecular contrast agent. After the other two thirds of the slides were fixed, half of them were stained with Thionine to identify the tumor, and the other half were stained with anti-von Willebrand Factor viii antibody to demonstrate the tumor-associated neovascularization.

\subsection{Spectroscopic photoacoustic tomography experimental setup}

The experimental setup for the in vivo spectroscopic photoacoustic tomography of a nude mouse head is shown in Fig. 1. A tuneable Ti:Sa nanosecond pulse laser (LT-2211A, Lotis T II, Minsk, Belarus) pumped by an Nd:YAG laser (LS-2137/2, Lotis T II, Minsk, Belarus) was employed to provide multiple-wavelength laser pulses with a pulse repetition rate of $10 \mathrm{~Hz}$. The laser beam was expanded by a concave lens, homogenized by a light diffuser, and then delivered to the animal head. The incident energy density of the laser beam on the surface of the mouse head was controlled at $\sim 20 \mathrm{~mJ} / \mathrm{cm}^{2}$ (within the ANSI standard). The laser energy of each single laser was monitored and recorded using a photodiode (PD), and then the recorded PD signals were used for compensation of the laser energy fluctuations. In this project, two types of ultrasonic transducers (V323/2.25MHz, XMS-310/10MHz, Panametrics) were used as detectors to receive the induced acoustic signals. The diameters of the active areas are $6 \mathrm{~mm}$ for the $2.25 \mathrm{MHz}$ transducer and $2 \mathrm{~mm}$ for the $10 \mathrm{MHz}$ transducer. The nominal bandwidths for the $2.25 \mathrm{MHz}$ and $10 \mathrm{MHz}$ transducers are $66 \%$ and $80 \%$, respectively.

A computer controlled step motor drove the $2.25 \mathrm{MHz}$ transducer to circularly scan the nude mouse brain at a 
depth of $\sim 3 \mathrm{~mm}$ below the scalp surface with a scanning radius of $4.5 \mathrm{~cm}$ (note that the depth of the $2.25 \mathrm{MHz}$ transducer is the depth of the tumor inoculation). On the other hand, the $10 \mathrm{MHz}$ transducer was driven to circularly scan at a depth similar to that of the scalp surface with a scanning radius of $3.5 \mathrm{~cm}$ for acquiring the structural imaging of the brain cortex. The scanning step size used here is 3 degrees. The mouse was fixed by a homemade restraining mount with its head protruding into the water tank through a hole in the bottom. The hole was sealed with a piece of polyethylene membrane. The mouse head surface was covered with a thin layer of ultrasonic coupling gel. The detected photoacoustic signals were amplified and then digitized by an oscilloscope. The digitized signals were transferred to a computer, and the distribution of the optical absorption in the imaging plane (x-y plane) was reconstructed using a modified back-projection algorithm after a full view scanning. For spectroscopic PAT, the wavelengths were changed at each scanning step. Note that four wavelengths, $764 \mathrm{~nm}, 784 \mathrm{~nm}, 804 \mathrm{~nm}$ and $824 \mathrm{~nm}$, were used in this study.

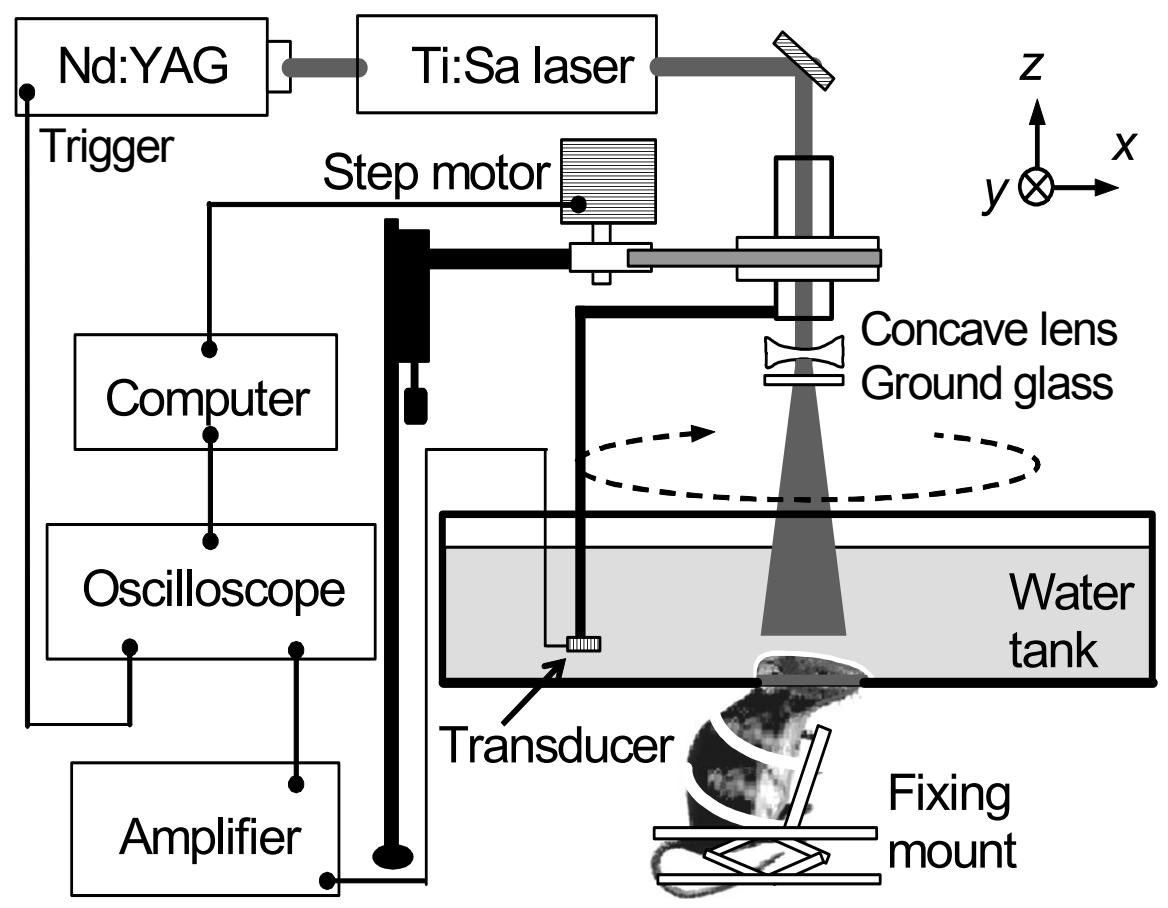

Figure 1. Experimental setup of in vivo spectroscopic PAT for nude mouse brain imaging.

\subsection{Spectroscopic photoacoustic molecular imaging and oxygen saturation imaging}

The spectroscopic PAT offers the opportunity to separate the contributions from different absorbers based on the differences in the optical absorption spectra among the absorbers. With the administration of the NIR molecular contrast agent, we can assume that there are only three main absorbers (the contrast agent, $\mathrm{HHb}$ and $\mathrm{O}_{2} \mathrm{Hb}$ ) in the imaged brain tissue in the NIR range. Therefore, the tissue absorption coefficient at wavelength $\lambda_{i}$ can be calculated as

$\mu_{a}\left(\lambda_{i}\right)=k \varepsilon_{\mathrm{HHb}}\left(\lambda_{i}\right)[\mathrm{HHb}]+k \varepsilon_{\mathrm{O}_{2} \mathrm{Hb}}\left(\lambda_{i}\right)\left[\mathrm{O}_{2} \mathrm{Hb}\right]+k \varepsilon_{\text {ContrastAgent }}\left(\lambda_{i}\right)[$ ContrastAgent $]$,

where $\varepsilon_{\mathrm{HHb}}\left(\lambda_{i}\right), \varepsilon_{\mathrm{O}_{2} \mathrm{Hb}}\left(\lambda_{i}\right)$ and $\varepsilon_{\text {ContrastAgent }}\left(\lambda_{i}\right)$ are the known molar extinction coefficients of $\mathrm{HHb}, \mathrm{O}_{2} \mathrm{Hb}$ and the contrast agent at wavelength $\lambda_{i}$, respectively; ${ }^{9}[\mathrm{HHb}], \quad\left[\mathrm{O}_{2} \mathrm{Hb}\right]$ and $[$ ContrastAgent $]$ are the molar concentrations of hemoglobin, oxy-hemoglobin and the molecular contrast agent, respectively. Since the reconstructed 
photoacoustic image $P A\left(\lambda_{i}\right)$ is proportional to the absorption coefficient after being normalized by the estimated tissue's optical wavelength-dependent attenuation and the incident laser fluence, the $\mu_{a}\left(\lambda_{i}\right)$ in Equation (1) can be replaced by $P A\left(\lambda_{i}\right)$. Using multiple wavelength measurements and known molar extinction coefficients, point-bypoint concentration distributions of the molecular contrast agent, $\mathrm{HHb}$ and $\mathrm{O}_{2} \mathrm{Hb}$ can be estimated by solving the set of linear equations from (1) using standard linear least-squares methods. Using estimated concentrations of $\mathrm{HHb}$ and $\mathrm{O}_{2} \mathrm{Hb}$, a point-by-point blood oxygen saturation $\left(\mathrm{SO}_{2}\right)$ image can be calculated according to the following equation:

$\mathrm{SO}_{2}=\frac{\left[\mathrm{O}_{2} \mathrm{Hb}\right]}{\left[\mathrm{O}_{2} \mathrm{Hb}\right]+[\mathrm{HHb}]}$

\section{RESULTS}

Fig. 2(A) shows a photograph of a nude mouse brain with a U87 glioblastoma tumor, in which the tumor foci can be seen at the right side of the brain. Figs. 2 (B) is a Thionine stained horizontal section photo of the nude mouse brain with a U87 tumor at a depth of $\sim 2 \mathrm{~mm}$ from the brain cortex. This histological staining section shows the U87 glioblastoma tumor located at a depth of $\sim 2 \mathrm{~mm}$ from the brain cortex. The integrin $\alpha_{v} \beta_{3}$-targeted peptide-ICG conjugated NIR fluorescent contrast agent was injected intravenously through the nude mice tail 24 hours before the spectroscopic PAT imaging. The contrast agent bonded to the $\alpha_{v} \beta_{3}$ integrin which is over-expressed by newly formed tumor microvessels. An In vivo fluorescent image (Fig. 2 (C)) was acquired on the same nude mouse before the PAT experiments to verify the molecular contrast agent uptake and compare with the spectroscopic PAT images. The fluorescence image in Fig. 2 (C) indicates the uptake of the contrast agent in the mouse brain. Fig. 2 (D) is the fluorescence frozen section image of the brain acquired by the Odyssey fluorescence microscanner, which further verifies the uptake of the contrast agent and the depth of the U87 tumor. Fig. 2 (E) shows spectroscopic PAT based molecular imaging showing the contrast agent distribution in the brain. In Fig. 2(E), it is clearly shown that the contrast agent is mainly accumulated at the tumor foci. Since PAT overcomes the resolution disadvantage of optical imaging that is due to the overwhelming light scattering in biological tissue, the resolution of the PAT image (Fig. 2 (E)) is higher than the planar fluorescence image (Fig. 2(C)). Hence, with the help of the molecular contrast agent, the tumor position is more precisely localized in Fig. 2 (E) than that in Fig. 2(C). In addition, tumor hypoxia, which is an important parameter in tumor therapy, was also imaged by the spectroscopic PAT, as shown in the blood oxygenation image in Fig. 2 (F).. Obviously, the level of blood oxgen saturation in the tumor foci is lower than that in the normal brain tissue. From Fig. 2, we can conclude that spectroscopic PAT can assess point-by-point functional parameters and changes in tumor areas in the brain quantitatively with high spatial resolution and, therefore, will provide better understanding of tumor biology and possible treatment.

\section{CONCLUSION}

The results which we presented here demonstrate that spectroscopic PAT-based molecular imaging of $\alpha v \beta_{3}$ integrin was successfully achieved by using the $\alpha v \beta_{3}$-targeted NIR contrast agent. According to the relative contrast agent concentrations measured by spectroscopic PAT and the total injection dosage, we can quantify relatively the corresponding $\alpha v \beta_{3}$ integrin distribution inside the nude mouse brain. This will improve our understanding of tumor angiogenesis at the cellular level since $\alpha_{v} \beta_{3}$ integrin is responsible for facilitating the spread of new blood vessels. Eventually these insights will lead to better understanding of tumor biology and new approaches for tumor therapy. In the meantime, tumor hypoxia imaging can also be provided by the spectroscopic PAT. Tumor hypoxia is very important in tumor biology and therapy. Hypoxia stimulates the tumor cells' production of acids; the hypoxia and acidity of tumors have important consequences for anti-tumor therapy and can contribute to the progression of tumors to a more aggressive metastatic phenotype. ${ }^{10,11,12}$ In theory, any type of pathological reaction can be monitored at the cellular level by spectroscopic PAT-based molecular imaging if the proper spectroscopic PAT targeting contrast agents for the chemicals responsible for the reaction can be employed. In the near future, spectroscopic PAT will play an important role in molecular imaging in tumor-related researches. 


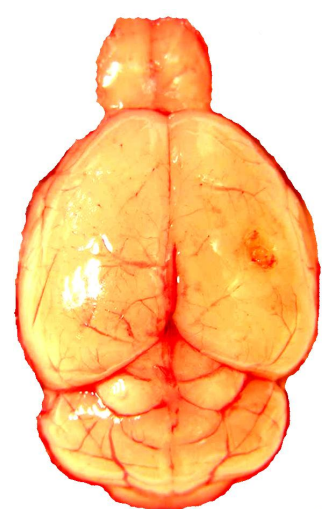

(A)

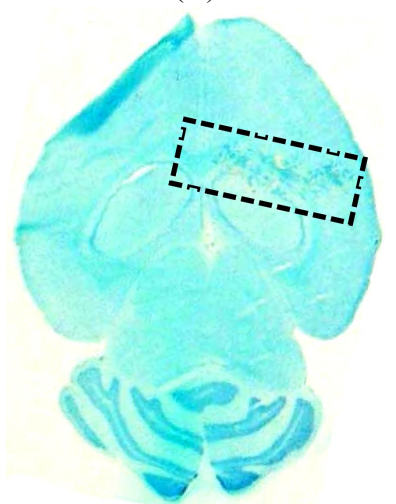

(B)

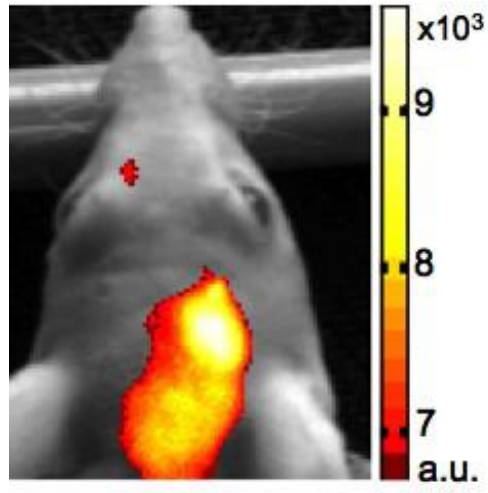

(C)

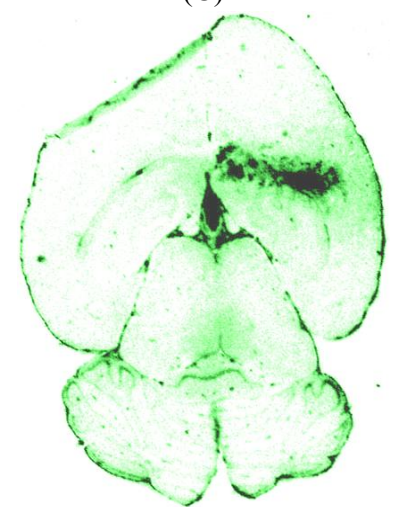

(D)

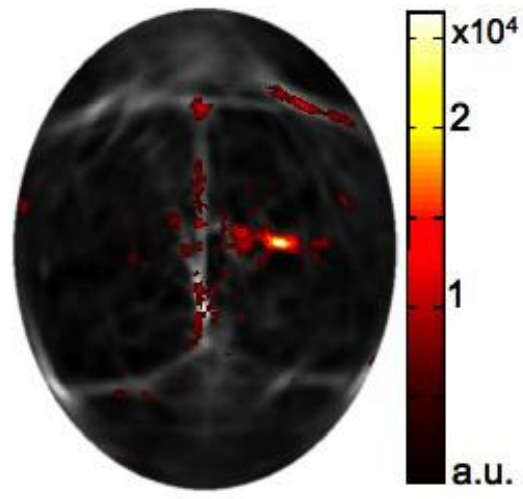

(E)

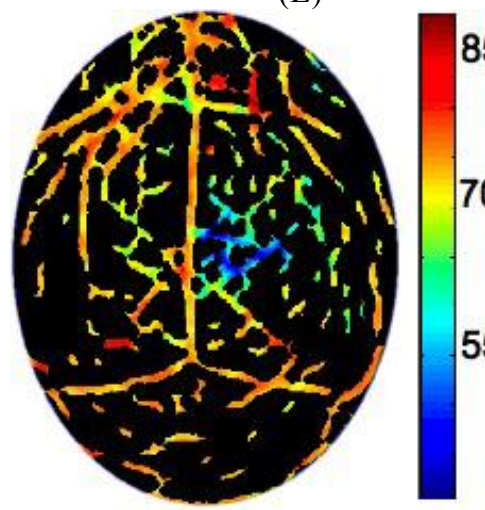

(F)

Figure 2. (A) Photography of a nude mouse brain with U87 glioblastoma tumor; (B) Thionine stained horizontal section photo of the nude mouse brain with U87 tumor; (C) Fluorescence image of the nude mouse head superimposed on the planar image; (D) Fluorescence frozen section image of the brain acquired by Odyssey fluorescence microscanner; (E) Estimated distribution of the molecular contrast agent in the brain superimposed on the photoacoustic brain structure image; (F) Blood oxygen saturation image of nude mice brain with U87 tumor; Both (E) and (F) are acquired by noninvasive in vivo spectroscopic PAT; (B) and (D) are at the depth of $\sim 2 \mathrm{~mm}$ from the brain cortex; the dotted area indicates the tumor position.

\section{ACKNOWLEDGMENTS}

This project was sponsored in part by the National Institute of Health grants R01 EB000712 and R01 NS46214. L. V. Wang's email is lwang@tamu.edu.

\section{REFERENSES}

${ }^{1}$ Weissleder, R., Reimer, P., Lee, A. S., et al, "MR receptor imaging: ultrasmall iron oxide particles targeted to asialoglycoprotein receptors", Am. J. Roentgenol (155), 1161-1167, 1990.

${ }^{2}$ Tjuvajev, J. G., Stockhammer, G., Desai, R., et al, "Imaging the expression of transfected genes in vivo", Cancer Res. (55), 6126-6132, 1995.

${ }^{3}$ Mayerhofer, R., Araki, K., and Szalay, A. A., "Monitoring of spatial expression of firefly luciferase in transformed zebrafish", J. Biolumin. Chemilumin (10), 271-275, 1995.

${ }^{4}$ T. F. Massoud and S. S. Gambhir, “ Molecular imaging in the living subjects: seeing fundamental biological processes in a new light”, Genes and Development 17, 545-580, 2003. 
${ }^{5}$ X. Wang, G. Ku, M. A. Wegiel, et al, "Non-invasive photoacoustic angiography of animal brains in vivo with NIR light and an optical contrast agent", Optics Letters 29 (7), 730-732, 2004.

${ }^{6} \mathrm{X}$. Wang, Y. Pang, G. Ku, et al, "Non-invasive laser-induced photoacoustic tomography for structural and functional imaging of the brain in vivo," Nature Biotechnology 21 (7), 803-806, 2003.

${ }^{7}$ Wei Wang et al., "Near-infrared optical imaging of integrin $\alpha v \beta 3$ in human tumor xenografts," Molecular Imaging 3 (4), 1-9, 2004.

${ }^{8}$ S. LAL et al., "An implantable guide-screw system for brain tumor studies in small animals," J. Neurosurg 92, 326333, 2000.

${ }_{9}$ W. G. Zijlstra, A. Buursma, O. W. van Assendelft, "Visible and near infrared absorption spectra of human and animal hemoglobin, determination and application", VSP, The Netherlands, 2000.

${ }^{10}$ P. Vaupel ,M. Hockel, "Tumor oxygenation and its relevance to tumor physiology and treatment", Adv. Exp. Med. Biol. (510) 45-49, 2003.

11 G.U. Dachs ,G.M. Tozer, "Hypoxia modulated gene expression: angiogenesis, metastasis and therapeutic exploitation", Eur. J. Cancer (36) 1649-1660, 2000.

12 G.L. Semenza, "HIF-1: mediator of physiological and pathophysiological responses to hypoxia", J. Appl. Physiol. (88) 1474-1480, 2000. 